\title{
Simulation of flow in two-sided lid-driven square cavities by the lattice Boltzmann method
}

\author{
D. Arumuga Perumal \& A. K. Dass \\ Department of Mechanical Engineering, \\ Indian Institute of Technology Guwahati, Guwahati 781039, India
}

\begin{abstract}
Due to the presence of corner eddies that change in number and pattern, the classical one-sided lid-driven cavity problem has been found to be particularly suitable to study various aspects of the performance of solution algorithms for incompressible viscous flows. More recently, the flow induced by the motion of two facing walls (two-sided lid-driven cavity) has also been investigated by Kuhlmann et al. For some aspect ratios this study demonstrates the existence of a multiplicity of solutions. However, for the aspect ratio of unity no multiplicity of solutions has been observed. Also it is found that for parallel motion of the walls, there appears a pair of counter-rotating secondary vortices of equal size near the centre of a wall. Because of symmetry, this pair of counter-rotating vortices has similar shapes and their detailed study as to how they grow with increasing Reynolds number has not yet been made. Such a study is attempted in this paper through the lattice Boltzmann method (LBM), as the problem has the potential of being used for testing various solution methods for incompressible viscous flows. The results for the antiparallel motion of the walls are also presented in detail. As the problem has not been investigated before, to lend credibility to the results they are further compared with those obtained from a finite difference method (FDM) code developed for this purpose.
\end{abstract}

Keywords: two-sided lid-driven cavity, lattice Boltzmann method, finite difference method, D2Q9 model, bounce-back boundary condition.

\section{Introduction}

A number of experimental and numerical studies have been conducted to investigate the flow field of a lid-driven cavity flow in recent decades. Ghia et al. 
[1] have applied a multi-grid strategy and presented solutions for Reynolds numbers starting from $\mathrm{Re}=100$ to as high as $\mathrm{Re}=10000$. A review on computational and also experimental studies on lid-driven cavity flow can be found in Shankar and Deshpande [2]. They have studied and analyzed corner eddies, nonuniqueness, transition and turbulence in the lid-driven cavity. Kuhlmann et al. [3] have done several experiments on a two-sided lid-driven cavity with various spanwise aspect ratios. Blohm and Kuhlmann [4] experimentally investigated the incompressible fluid flow in a rectangular container driven by two facing sidewalls that move steadily in an anti-parallel direction up to Reynolds number 1200.

Albensoeder et al. [5] were the first to investigate the nonlinear regime and found multiple two-dimensional states. They have found seven and five flow states in antiparallel and parallel motion respectively. Kalita et al. [6] developed an HOC algorithm for Stream-function vorticity formulation of the $2 \mathrm{D} \mathrm{N}-\mathrm{S}$ equations on graded Cartesian meshes. They used the algorithm to compute the flow in a two-sided 2D lid-driven cavity [7] where, besides wall shear, free shear flow is also encountered.

Many researchers [9] carried out simulations of one-sided lid-driven cavity flow by lattice Boltzmann method. Yong G Lai et al. [10] compared the lattice Boltzmann method and the finite volume Navier-Stokes solver and concluded that bounce-back boundary condition has better than first order accuracy. The present work uses lattice Boltzmann BGK model (LBGK) with single time relaxation and bounce-back boundary condition to investigate the flow driven by parallel and antiparallel motion of two facing walls in a square cavity for Reynolds number up to 2000. A nine-bit square lattice incompressible LB model in $2 \mathrm{D}$ space has been used in the present work since it is known to give more accurate results compared to seven-bit incompressible LB model.

The rest of the paper is organized as follows. In Section 2, LBGK with single time relaxation and $2 \mathrm{D}$ nine-velocity lattice model is defined. The numerical procedures for LBM and stream function-vorticity based FDM is also described in this section. In Section 3, the two-sided lid-driven cavity problem is described and the results from LBM simulation with parallel and antiparallel motion of the walls are presented. The results are discussed in Section 4. Based on the results obtained concluding remarks are made in Section 5.

\section{Numerical methods}

\subsection{Lattice Boltzmann method}

The lattice Boltzmann method represents an alternative possibility for the direct simulation of the incompressible flow. It has been shown that the accuracy of the lattice Boltzmann method is of second order both in space and time [9]. The lattice Boltzmann equation, which can be linked to the Boltzmann equation in kinetic theory, is formulated as [8]

$$
f_{i}\left(\mathbf{x}+c_{i} \Delta t, t+\Delta t\right)-f_{i}(\mathbf{x}, t)=\Omega_{i}
$$

where $f_{i}$ is the particle distribution function, $c_{i}$ is the particle velocity along the ith direction and $\Omega_{i}$ is the collision operator. The so-called lattice BGK model 
with single time relaxation, which is a commonly used lattice Boltzmann method, is given by

$$
f_{i}\left(\mathbf{x}+c_{i} \Delta t, t+\Delta t\right)-f_{i}(\mathbf{x}, t)=-\frac{1}{\tau}\left(f_{i}(\mathbf{x}, t)-f_{i}^{(0)}(\mathbf{x}, t)\right)
$$

Here $f_{i}^{(0)}(\mathbf{x}, t)$ is the equilibrium distribution function at $\mathbf{x}, \mathbf{t}$ and $\tau$ is the time relaxation parameter.

The D2Q9 square lattice used here has nine discrete velocities. A square lattice with unit spacing is used on each node with eight neighbours connected by eight links. Particles residing on a node move to their nearest neighbours along these links in unit time step. The occupation of the rest particle is defined as $f_{0}$. The occupation of the particles moving along the axes are defined as $f_{1}, f_{2}$, $f_{3}, f_{4}$, while the occupation of diagonally moving particles are defined as $f_{5}, f_{6}$, $f_{7}, f_{8}$. The particle velocities are defined as

$$
\begin{aligned}
& \mathrm{c}_{i}=0, \quad i=0 \\
& \mathrm{c}_{i}=(\cos (\pi / 4(i-1)), \sin (\pi / 4(i-1))), \quad i=1,2,3,4 \\
& \mathrm{c}_{i}=\sqrt{2}(\cos (\pi / 4(i-1)), \sin (\pi / 4(i-1))), \quad i=5,6,7,8 .
\end{aligned}
$$

The macroscopic quantities such as density $\rho$ and momentum density $\rho u$ are defined as velocity moments of the distribution function $f_{i}$ as follows:

$$
\begin{gathered}
\rho=\sum_{i=0}^{N} f_{i}, \\
\rho u=\sum_{i=0}^{N} f_{i} c_{i} .
\end{gathered}
$$

The density is determined from the particle distribution function. The density and the velocities satisfy the Navier-Stokes equations in the low-Mach number limit by using the Chapman-Enskog expansion [8]. In the nine-velocity square lattice, a suitable equilibrium distribution function has been proposed [9] with,

$$
\begin{aligned}
& f_{i}^{(0)}=\frac{4}{9} \rho\left[1-\frac{3}{2} u^{2}\right], \quad i=0 \\
& f_{i}^{(0)}=\frac{1}{9} \rho\left[1+3\left(c_{i} \cdot u\right)+4.5\left(c_{i} \cdot u\right)^{2}-1.5 u^{2}\right], \quad i=1,2,3,4 \\
& f_{i}^{(0)}=\frac{1}{36} \rho\left[1+3\left(c_{i} \cdot u\right)+4.5\left(c_{i} \cdot u\right)^{2}-1.5 u^{2}\right], \quad i=5,6,7,8
\end{aligned}
$$

where the lattice weights are $w_{0}=4 / 9, w_{1}=w_{2}=w_{3}=w_{4}=1 / 9$ and $w_{5}=w_{6}=w_{7}=w_{8}=1 / 36$. The relaxation time $\tau$ is related to the viscosity by

$$
\tau=\frac{6 v-1}{2}
$$

where $v$ is the kinematic viscosity. It was seen that $\tau=0.5$ is the critical value for ensuring a non-negative kinematic viscosity. Numerical instability can be expected for $\tau$ close to this critical value. This situation takes place at high Reynolds numbers. In this work Reynolds numbers up to 2000 in a lattice size of $257^{2}$ have been investigated.

In LBM several boundary conditions have been proposed [10]. The bounceback scheme was used in these simulations to copy the velocity no-slip condition 
on walls. In this scheme, the particle distribution function at the wall lattice node is assigned to be the particle distribution function of its opposite direction. The basic argument for the use of 'on-grid bounce-back model' is that it is both mathematically applicable and quite relevant for LBE simulations of fluid flows in simple bounded domains. For this reason, this boundary condition has been employed here on the two stationary walls. However for the moving walls, the equilibrium boundary condition is applied [9]. At the lattice nodes on the moving walls, flow-variables are re-set to their pre-assumed values at the end of every streaming-step. A lid-velocity of $U=0.1$ has been considered in this work.

\subsubsection{Numerical algorithm}

The velocities at all nodes except those on the moving walls are assumed to be zero at the time of starting the simulation. Initially, the equilibrium distribution function that corresponds to the flow-variables is assumed as the unknown particle distribution function for all nodes at $t=0$. Uniform density $\rho=1.0$ is imposed initially.

The solution procedure of the LBM at each time step comprise streaming and collision step, application of boundary conditions, calculation of particle distribution function followed by calculation of macroscopic variables.

The lattice Boltzmann Equation (LBE) is solved in the solution domain subjected to the above initial and boundary conditions on a uniform $2 \mathrm{D}$ mesh. It is seen that the numerical algorithm of the LBM is relatively simpler compared with conventional Navier-Stokes methods. Another benefit of the present approach is the easiness of programming.

\subsection{Finite difference (FD) stream function-vorticity based solver}

As the LBM method is intended to be used to compute an unexplored problem, need was felt to develop a finite difference code to attack the same problem thus providing a basis for comparison. The FD code numerically solves the 2D Navier-Stokes equation in stream function-vorticity formulation given by

$$
\begin{gathered}
\frac{\partial^{2} \psi}{\partial x^{2}}+\frac{\partial^{2} \psi}{\partial y^{2}}=-\omega \\
\frac{\partial \omega}{\partial \mathrm{t}}+u \frac{\partial \omega}{\partial x}+v \frac{\partial \omega}{\partial y}=\frac{1}{R e}\left(\frac{\partial^{2} \omega}{\partial x^{2}}+\frac{\partial^{2} \omega}{\partial y^{2}}\right) .
\end{gathered}
$$

In the code all space derivatives are centrally differenced and ADI method is used for time integration to the steady state. This code will provide a legitimate basis for comparison only after it has been validated and this exercise is presented in the next sub-section.

\subsection{Code validation}

The developed FD code is used to compute the single lid-driven flow in a square cavity on a $129^{2}$ grid. Well established results computed by Ghia et al. [1] exist for the same problem on a similar grid and this work is used for validating the present FD code. Figure 1 shows the steady-state u-velocity profile along a 
vertical line passing through the geometric centre of the cavity at $\mathrm{Re}=1000$. Here the top lid moves from left to right and it is observed that the agreement between our FD results and those of Ghia et al. [1] is excellent. The same figure also displays our LBM results, which again are in excellent agreement with the two results described earlier. In the next section the LBM results for the unexplored two-sided lid-driven cavity flow will be presented and the credibility of these results will be established through comparison with the results of the FD code.

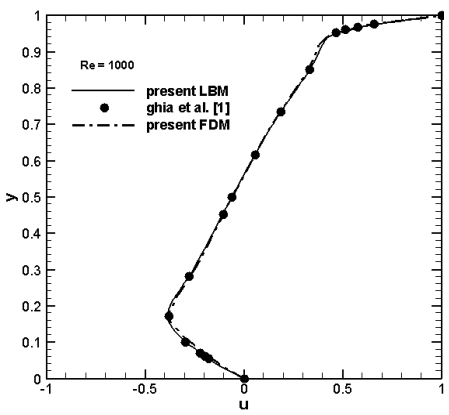

Figure 1: u-velocity profile at the vertical centreline for single lid-driven square cavity flow $(\mathrm{Re}=1000)$.

\section{Two-sided lid-driven cavity flow}

An incompressible viscous flow in a square cavity whose top and bottom walls move in the same (parallel motion) or opposite (antiparallel motion) direction with a uniform velocity is the problem investigated in the present paper. In the case of parallel wall motion, a free shear layer exists midway between the top and bottom walls apart from the wall bounded shear layers whereas in the case of antiparallel wall motion, only wall bounded shear layers exist.

\subsection{Parallel wall motion}

LBM results on a lattice size of $257 \times 257$ for the parallel wall motion are shown in Figure 2. When both the walls, say, the top and the bottom move in the same direction with the same velocity, the streamlines are found to be symmetric with respect to a line parallel to these walls and passing through the cavity centre. Figure 2(a) shows the streamline pattern for the parallel wall motion at $\mathrm{Re}=100$ with the top and bottom walls moving from left to right. Two counter-rotating primary vortices symmetric to each other are seen to form with a free shear layer in between. At this Reynolds number the primary vortex cores are seen to be somewhat away from the centres of the top and bottom halves of the lid-driven cavity towards the right-hand top and right-hand bottom corners respectively. At $\mathrm{Re}=400$ (Figure 2(b)), apart from the primary vortices two counter-rotating secondary vortices symmetrically placed about the horizontal centreline are seen to appear near the centre of the right wall. Figures 2(c) and 2(d) show the 
50 Advances in Fluid Mechanics VII

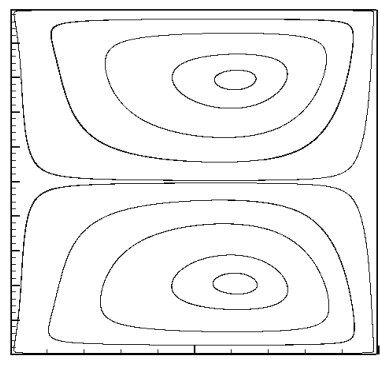

(a) $\operatorname{Re}=100$

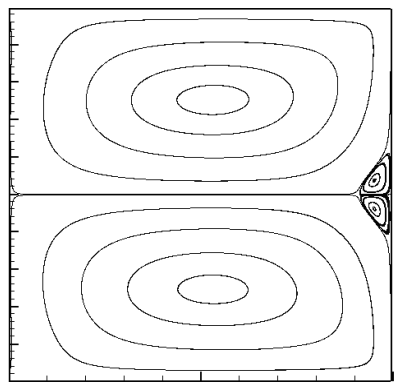

(c) $\operatorname{Re}=1000$

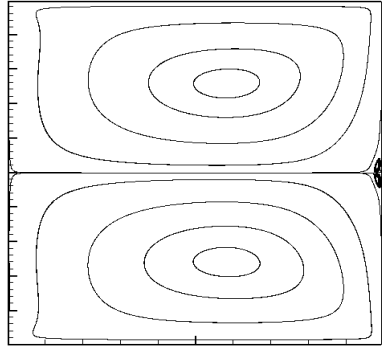

(b) $\operatorname{Re}=400$

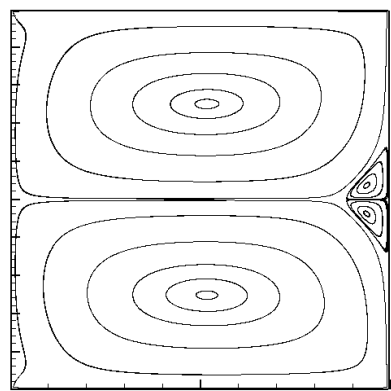

(d) $\operatorname{Re}=2000$

Figure 2: $\quad$ Streamline pattern for parallel wall motion at (a) $\operatorname{Re}=100$ (b) $\operatorname{Re}=$ 400 (c) $\mathrm{Re}=1000$ and (d) $\mathrm{Re}=2000$ by LBM on a $257^{2}$ lattice.

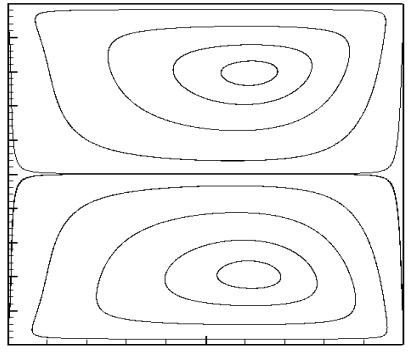

(a) $\operatorname{Re}=100$

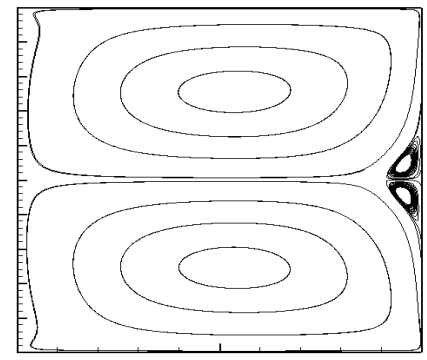

(c) $\operatorname{Re}=1000$

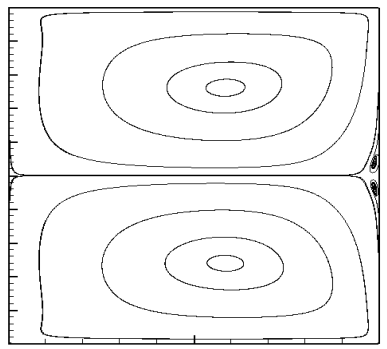

(b) $\operatorname{Re}=400$

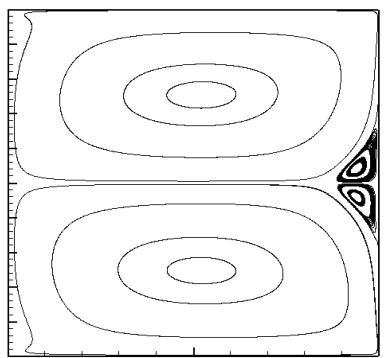

(d) $\mathrm{Re}=2000$

Figure 3: Streamline pattern for parallel wall motion at (a) $\operatorname{Re}=100$ (b) $\operatorname{Re}=$ 400 (c) $R e=1000$ and (d) $R e=2000$ by FDM on a $129^{2}$ grid. 
streamline patterns at $\mathrm{Re}=1000$ and $\mathrm{Re}=2000$ respectively. From Figure 2 it is observed that as the Reynolds number increases the cores of the primary vortices move from near the top right and bottom right corners to the centres of the top and bottom halves of the cavity respectively. Also as the Reynolds number increases the secondary vortices near the centre of the right wall grow in size. The counter-rotating pairs of primary and secondary vortices maintain their centro-symmetry for all the Reynolds number investigated in this work. As mentioned earlier these results are now substantiated by comparison with the results given by the validated FDM code. Figure 3 shows the FDM streamline patterns on a $129^{2}$ grid for $\mathrm{Re}=100,400,1000$ and 2000 as before. Comparison with Figure 2 shows that LBM streamline patterns compare very well with those for the FDM. Figure 4(a) shows the comparison for horizontal velocity along the vertical centreline of the cavity and Figure 4(b) shows the comparison of vertical velocity on a horizontal line at a height of three quarters from the bottom wall. Agreement of the velocity profiles given by both methods is again excellent. Table 1 gives the locations of the vortices given by LBM and FDM for $\mathrm{Re}=100$, $400,1000,1500$ and 2000. All these results show that the agreement is very good thus lending credibility to the results for these unexplored problems.

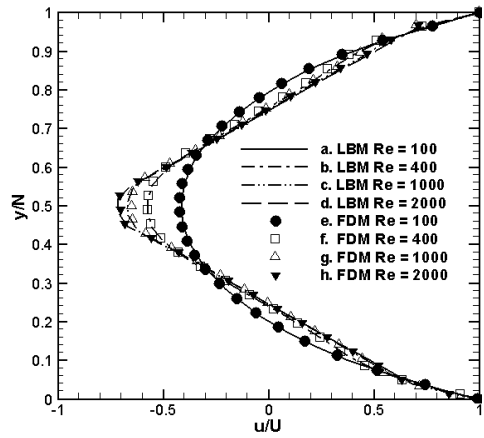

(a)

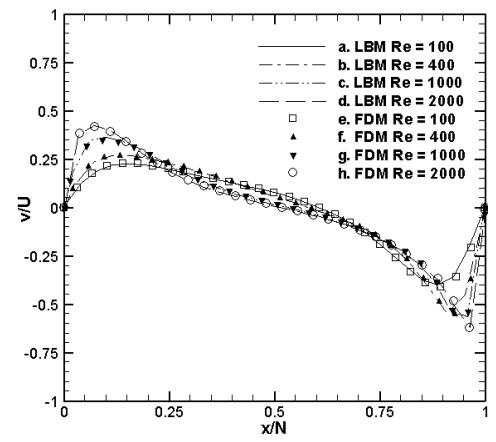

(b)

Figure 4: $\quad$ For parallel wall motion (a) u-velocity along vertical line passing through $\mathrm{x}=0.50$ (b) v-velocity along horizontal line passing through $\mathrm{y}=0.75$.

\subsection{Antiparallel wall motion}

LBM results on a lattice size of $257^{2}$ for the antiparallel wall motion are shown in Figure 5. When both the walls move in opposite direction with the same velocity, top to the right and bottom to the left in this paper, a single primary vortex centred at the geometric centre of the cavity is formed (Figures 5(a) and $5(\mathrm{~b})$ ). These two figures show the streamline patterns for $\mathrm{Re}=100$ and 400 respectively. Figures 5(c) and 5(d) show the streamline patterns for $\operatorname{Re}=1000$ and 2000, which show the appearance of also two secondary vortices near the top left and the bottom right corners of the cavity. It may be noted that the corresponding vortex for a single lid-driven cavity flow does not appear at a Reynolds number as low as 1000 but much later (i.e. at a higher value beyond 


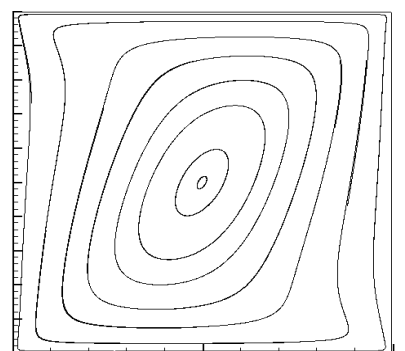

(a) $\operatorname{Re}=100$

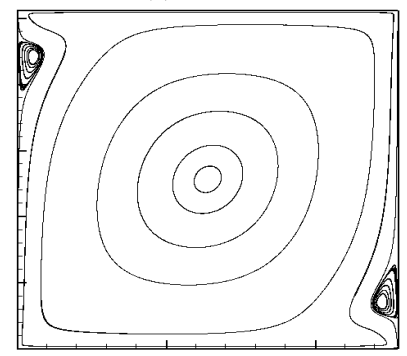

(c) $\operatorname{Re}=1000$

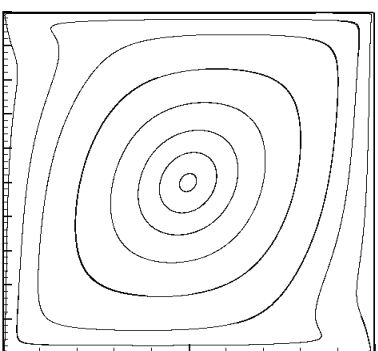

(b) $\mathrm{Re}=400$

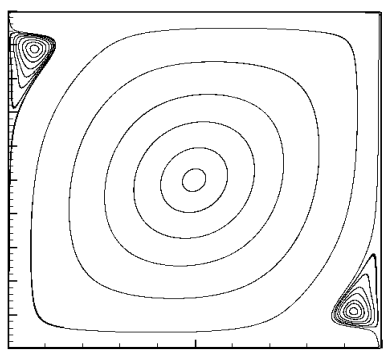

(d) $\operatorname{Re}=2000$

Figure 5: $\quad$ Streamline pattern for antiparallel wall motion at (a) $\mathrm{Re}=100$ (b) $\mathrm{Re}=400$ (c) $\mathrm{Re}=1000$ and (d) $\mathrm{Re}=2000$ by $\mathrm{LBM}$ on a $257^{2}$ lattice.

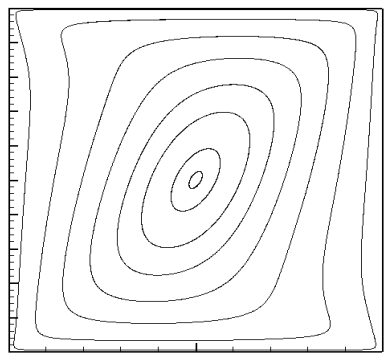

(a) $\operatorname{Re}=100$

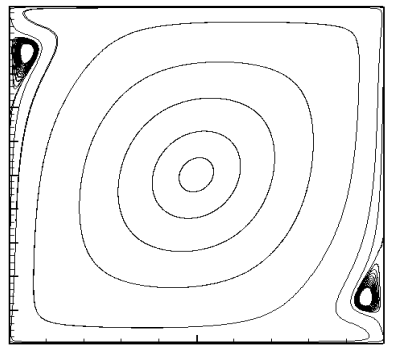

(c) $\operatorname{Re}=1000$

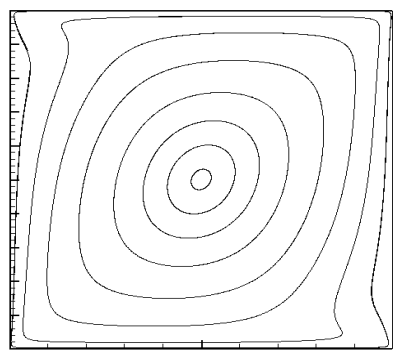

(b) $\operatorname{Re}=400$

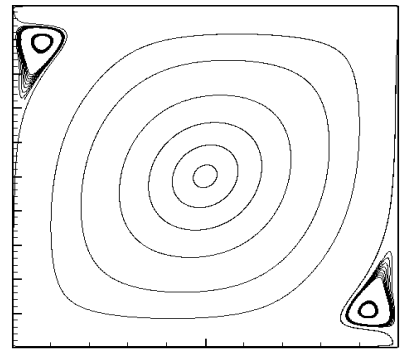

(d) $\operatorname{Re}=2000$

Figure 6: $\quad$ Streamline pattern for antiparallel wall motion at (a) $\operatorname{Re}=100$ (b) $\mathrm{Re}=400$ (c) $\mathrm{Re}=1000$ and (d) $\mathrm{Re}=2000$ by FDM on a $129^{2}$ lattice. 


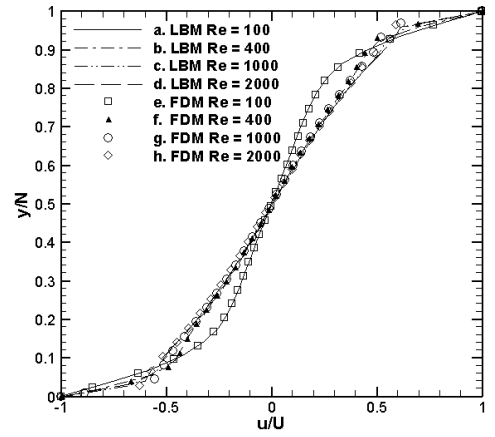

(a)

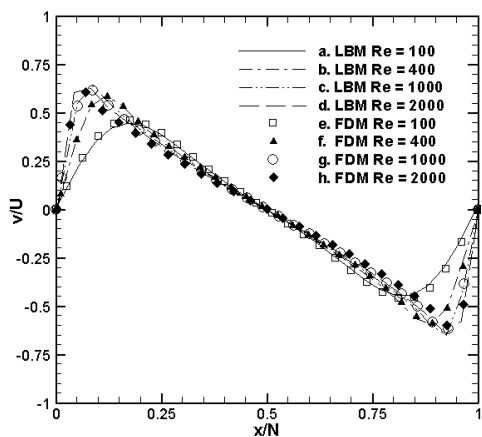

(b)

Figure 7: For antiparallel wall motion (a) u-velocity along vertical line passing through $\mathrm{x}=0.50$ (b) v-velocity along horizontal line passing through $\mathrm{y}=0.50$.

Table 1: Locations of the vortices for parallel wall motion: a. FDM, b. LBM.

\begin{tabular}{|c|c|c|c|c|c|c|c|c|}
\hline \multirow{3}{*}{$\mathrm{Re}$} & \multicolumn{4}{|c|}{ Primary vortex centres } & \multicolumn{4}{|c|}{ Secondary vortex centres } \\
\hline & \multicolumn{2}{|c|}{ Bottom } & \multicolumn{2}{|c|}{ Top } & \multicolumn{2}{|c|}{ Bottom } & \multicolumn{2}{|c|}{ Top } \\
\hline & $\mathrm{x}$ & $\mathrm{y}$ & $\mathrm{x}$ & $\mathrm{y}$ & $\mathrm{x}$ & $\mathrm{y}$ & $\mathrm{x}$ & $\mathrm{y}$ \\
\hline \multirow{2}{*}{100} & a. 0.6146 & 0.2025 & 0.6146 & 0.7956 & $\ldots$ & $\ldots$ & $\ldots$ & $\ldots$ \\
\hline & b. 0.6145 & 0.2024 & 0.6145 & 0.7949 & $\ldots$ & $\ldots$ & $\ldots$ & $\ldots$ \\
\hline \multirow{2}{*}{400} & a. 0.5844 & 0.2387 & 0.5844 & 0.7552 & 0.9872 & 0.4636 & 0.9872 & 0.5262 \\
\hline & b. 0.5845 & 0.2388 & 0.5845 & 0.7549 & 0.9875 & 0.4713 & 0.9874 & 0.5283 \\
\hline \multirow{2}{*}{1000} & a. 0.5352 & 0.2453 & 0.5352 & 0.755 & 0.9550 & 0.4572 & 0.9550 & 0.5408 \\
\hline & b. 0.5314 & 0.2431 & 0.5314 & 0.7556 & 0.9528 & 0.4619 & 0.9528 & 0.5365 \\
\hline \multirow{2}{*}{1500} & a. 0.5245 & 0.2453 & 0.5267 & 0.7528 & 0.9444 & 0.4572 & 0.9443 & 0.5429 \\
\hline & b. 0.5234 & 0.2434 & 0.5234 & 0.7518 & 0.9434 & 0.4569 & 0.9433 & 0.5385 \\
\hline \multirow{2}{*}{2000} & a. 0.5132 & 0.2474 & 0.5132 & 0.7528 & 0.9400 & 0.4573 & 0.9400 & 0.5478 \\
\hline & b. 0.5108 & 0.2489 & 0.5108 & 0.7497 & 0.9378 & 0.4598 & 0.9377 & 0.5389 \\
\hline
\end{tabular}

2000). It has also been observed that primary vortex centre remains at the geometric centre of the cavity even for these higher values of $\mathrm{Re}=1000$ and 2000. However the size of the secondary vortices are seen to increase between $\operatorname{Re}=1000$ and 2000. Similar increase in size of the secondary vortices with Reynolds numbers was also observed for the parallel wall motion. These results are now substantiated by comparison with the results of the validated FDM code. Figure 6 gives for the same configuration the FDM streamline patterns on a $129^{2}$ grid for $\mathrm{Re}=100,400,1000$ and 2000. Comparisons with Figure 5 shows that LBM streamline patterns compare very well with those for the FDM. Figures 7(a) and 7(b) show the comparison between LBM and FDM for the horizontal velocity along the vertical centreline and the vertical velocity along the horizontal centreline and the agreement is excellent once again. 
Table 2: $\quad$ Locations of the vortices for antiparallel motion: a. FDM, b. LBM.

\begin{tabular}{|c|c|c|c|c|c|c|}
\hline \multirow{3}{*}{$\mathrm{Re}$} & \multicolumn{2}{|c|}{ Primary Vortex (PV) } & \multicolumn{4}{|c|}{ Secondary Vortices (SV) } \\
\hline & \multirow[t]{2}{*}{$\mathrm{x}$} & \multirow[t]{2}{*}{$\mathrm{y}$} & \multicolumn{2}{|c|}{ Bottom Right } & \multicolumn{2}{|c|}{ Top Left } \\
\hline & & & $\mathrm{x}$ & $\mathrm{y}$ & $\mathrm{x}$ & $\mathrm{y}$ \\
\hline 100 & $\begin{array}{l}\text { a. } 0.4999 \\
\text { b. } 0.5002\end{array}$ & $\begin{array}{l}0.5001 \\
0.5001\end{array}$ & $\begin{array}{l}\cdots \\
\cdots\end{array}$ & $\begin{array}{l}\cdots \\
\cdots\end{array}$ & $\begin{array}{l}\cdots \\
\cdots\end{array}$ & $\begin{array}{l}\cdots \\
\ldots\end{array}$ \\
\hline 400 & $\begin{array}{l}\text { a. } 0.5002 \\
\text { b. } 0.5001\end{array}$ & $\begin{array}{l}0.4980 \\
0.4982\end{array}$ & $\begin{array}{l}\cdots \\
\ldots\end{array}$ & $\begin{array}{l}\cdots \\
\ldots\end{array}$ & $\begin{array}{l}\cdots \\
\cdots\end{array}$ & $\begin{array}{l}\cdots \\
\cdots\end{array}$ \\
\hline 1000 & $\begin{array}{l}\text { a. } 0.5007 \\
\text { b. } 0.5012\end{array}$ & $\begin{array}{l}0.4981 \\
0.4982\end{array}$ & $\begin{array}{l}0.9507 \\
0.9512\end{array}$ & $\begin{array}{l}0.1319 \\
0.1326\end{array}$ & $\begin{array}{l}0.0492 \\
0.0449 \\
\end{array}$ & $\begin{array}{l}0.8663 \\
0.8609 \\
\end{array}$ \\
\hline 1500 & $\begin{array}{l}\text { a. } 0.5005 \\
\text { b. } 0.5010\end{array}$ & $\begin{array}{l}0.4982 \\
0.4979\end{array}$ & $\begin{array}{l}0.9214 \\
0.9336\end{array}$ & $\begin{array}{l}0.1146 \\
0.1175\end{array}$ & $\begin{array}{l}0.0727 \\
0.0642\end{array}$ & $\begin{array}{l}0.8856 \\
0.8829\end{array}$ \\
\hline 2000 & $\begin{array}{l}\text { a. } 0.5003 \\
\text { b. } 0.5002\end{array}$ & $\begin{array}{l}0.5001 \\
0.4991\end{array}$ & $\begin{array}{l}0.9229 \\
0.9250\end{array}$ & $\begin{array}{l}0.1082 \\
0.1082\end{array}$ & $\begin{array}{l}0.0791 \\
0.0727\end{array}$ & $\begin{array}{l}0.8920 \\
0.8855\end{array}$ \\
\hline
\end{tabular}

Table 2 gives the locations of the vortices given by LBM and FDM for Re = $100,400,1000,1500$ and 2000 . It is seen that LBM results given by the figures and the table are in excellent agreement with the FDM results produced through the validated code. This lends credibility to the current LBM results for this unexplored problem.

\section{References}

[1] Ghia, U. Ghia, N. \& Shin, T., High-Re solutions for incompressible flow using N-S eqns and a multigrid method. J. Comp. Phys, 43, pp. 387-441, 1982.

[2] Shankar, P.N. \& Deshpande, M.D., Fluid Mechanics in the Driven Cavity. Ann. Rev. of Fluid Mech, 32, pp. 93-136, 2000.

[3] Kuhlmann, Wanschura, \& Rath, J., Flow in 2-sided lid-driven cavities: nonuniqueness, instability, cellular structures. J. F. Mech, 336 pp 267-299, 2001.

[4] Blohm, H. \& Kuhlmann, C., The two-sided lid-driven cavity: experiment on stationary and time-dependent flows. J. Fluid Mech, 450, pp. 67-95, 2002.

[5] Albensoeder, S. Kuhlmann, C. \& Rath J., Multiplicity of steady 2-D flow in two-sided lid-driven cavities. The Com. fluid Dym, 14, pp. 223-241, 2001.

[6] Kalita, J.C. Dass, A.K. \& Dalal, D., A trans.-free HOC scheme for steady conv-diff. on nonuniform grid. I. J. N. Me in Engg, 38, pp. 3497-3521, 2004.

[7] Kumar, N, Kalita, C, \& Dass, A.K., HOC computation of 2-Sided liddriven cavity flow. Int Con on Com. Mech \& Sim, Vol 2 B073, 1695-1701, 2006.

[8] He, X. \& Luo, L.S., Theory of the LBM: From the Boltzmann eqn to the lattice Boltzmann eqn. Phys. Rev. E, 56, pp. 6811-6817, 1997.

[9] Hou, S. Zou, Q. Chen, S. Doolen, G. \& Cogley, A.C., Simulation of Cavity Flow by the LBM. J. of Comput. Phys, 118, pp. 329-347, 1995.

[10] Lai, G. Lin, L. \& Huang, J., Accuracy and Efficiency study of a LBM for steady-state flow simulation. Num. Heat Transfer-B, 39, pp. 21-43, 2001. 